\title{
DIGITAL DS-CDMA RECEIVERS WORKING BELOW THE CHIP RATE
}

\author{
Irena Maravić‘ and Martin Vetterli ${ }^{\dagger \dagger}$ \\ ${ }^{\dagger}$ Audio-Visual Communications Laboratory \\ Swiss Federal Institute of Technology in Lausanne, CH-1015 Lausanne, Switzerland \\ ${ }^{\dagger \dagger}$ Department of EECS, University of California at Berkeley, Berkeley CA 94720, USA
}

\begin{abstract}
We consider the problem of designing low-complexity digital receivers for CDMA systems operating over channels with single or multiple propagation paths. We extend some of our recent sampling results for certain classes of nonbandlimited signals and develop a method that takes advantage of transform techniques to perform channel estimation and signal detection from a low-dimensional subspace of a received signal, that is, by sampling below the traditional Nyquist rate. By lowering the sampling rate we can reduce computational requirements compared to existing solutions, allow for slower A/D converters and significantly reduce power consumption of digital receivers. In effect, we use an algorithmic solution to improve hardware specifications in terms of complexity and power.
\end{abstract}

\section{INTRODUCTION}

Code-division multiple access (CDMA) is a widespread access protocol well suited for voice and data transmission, particularly over wireless communication networks. CDMA possesses several advantages over earlier access techniques due to a coded signal format and an expanded bandwidth. In particular, selective addressing capability, low-density power spectra and interference rejection are among properties that have prompted increased interest in CDMA as a flexible and spectrally efficient access strategy [10].

Although theoretically sound, implementation of CDMA receivers is not trivial in practice. On the one hand, in current attempts at low-power integrated radio solutions, a trend is to eliminate as much as possible the necessity for analog components and perform all processing digitally. On the other, application of proposed high-performance digital schemes [1] [5] [7] to real time systems is often not feasible, since most of them require high sampling rates (chip rate sampling or even fractional sampling) and therefore very fast and expensive analog-to-digital converters. Besides, sampling at the chip rate implies that all opera- tions are performed on an extensive set of samples, which makes such schemes computationally intense and often inefficient.

In this paper, we present a new approach toward designing low-complexity digital receivers for CDMA systems which uses lower than the Nyquist sampling rate and yet has performances similar to those of chip-sampled schemes. We extend some of our recent sampling results for certain classes of non-bandlimited signals [4] [9] to the problem of channel estimation and signal detection from a lowpass approximation of a received signal. Our approach leads to reduced computational requirements and lower power consumption compared to existing techniques, while providing similar performances.

\section{SUBSPACE CHANNEL ESTIMATION}

\subsection{Problem Formulation}

Consider the general case of a direct sequence (DS) CDMA system with $K$ users operating over a multipath fading channel with at most $L$ propagation paths for each user. We will assume that the channel varies slowly, i.e. it is considered constant over a channel estimation window. A common model for a received baseband signal $y(t)$ is

$$
y(t)=\sum_{n} \sum_{k=1}^{K} b_{k, n} \sum_{l=1}^{L} a_{k}^{(l)} s_{k}\left(t-\tau_{k}^{(l)}-n T_{s}\right)+\eta(t)
$$

where $s_{k}(t)$ is a signature sequence of length $N$ assigned to user $k, \tau_{k}^{(l)}$ denotes the delay of the signal of user $k$ along the $l$-th path, $a_{k}^{(l)}$ is a complex propagation coefficient, $b_{k, n}$ denotes the $n$-th bit sent by user $k, \eta(t)$ is additive white Gaussian noise and $T_{s}$ denotes a symbol duration.

The above model can be interpreted as saying that the received signal $y(t)$ is given by a sum of attenuated and delayed copies of transmitted signals and noise. Note that $y(t)$ has only $2 K L$ degrees of freedom (given knowledge of bits sent by each user), namely time delays $\tau_{k}^{(l)}$ and 
propagation coefficients $a_{k}^{(l)}$, which can be efficiently estimated if we consider the problem in the frequency domain.

Let $Y_{n}[m]$ denote DFT coefficients of the received signal $y(t)$

$$
Y_{n}[m]=\sum_{k=1}^{K} b_{k, n} \sum_{l=1}^{L} a_{k}^{(l)} S_{k}[m] e^{-j m \omega_{0} \tau_{k}^{(l)}}+N_{n}[m]
$$

where $\omega_{0}=2 \pi / T_{s}$ and $S_{k}[m]$ and $N_{n}[m]$ are DFT coefficients of signature sequences and noise respectively. A more compact form of (2) is given by

$$
Y_{r, n}[m]=\sum_{k=1}^{K} b_{k, n} c_{m k}+N_{n}[m]
$$

where $c_{m k}=S_{k}[m] \sum_{l=1}^{L}\left(a_{k}^{(l)} e^{-j m \omega_{0} \tau_{k}^{(l)}}\right)$, or equivalently,

$$
\mathbf{C} \cdot \mathbf{b}_{n}+\mathbf{N}_{n}=\mathbf{Y}_{r, n}
$$

Note that the coefficients $c_{m k}$ are given by a sum of complex exponentials, where the unknown time delays appear as complex frequencies while propagation coefficients appear as unknown weights. Therefore, the problem of channel estimation can be considered as a special case of classic spectral estimation problems [2] [6], where the parameters $\tau_{k}^{(l)}$ and $a_{k}^{(l)}$ can be uniquely found from $2 L$ coefficients $c_{m k}$, by applying either subspace methods for harmonic retrieval [6] or the annihilating filter approach [9]. A set of $2 L$ adjacent DFT coefficients $Y_{r, n}[m]$ used in (4) can be obtained, for example, from a set of uniform samples taken with the sinc kernel of bandwidth $\left[-L \omega_{0}, L \omega_{0}\right]$ [9].

The minimum required size of the matrix $\mathbf{C}$ is $2 L \times K$, thus in order to estimate $\mathbf{C}$ (given $\mathbf{b}_{n}$ and $\mathbf{Y}_{r, n}$ ) each user $k$ must send a training sequence $\mathbf{b}_{t k}$

$$
\mathbf{b}_{t k}=\left[\begin{array}{llll}
b_{t k, 1} & b_{t k, 2} & \ldots & b_{t k, K}
\end{array}\right]
$$

In the noiseless case, our method leads to a perfect estimation of the channel parameters by taking only $2 L$ samples per symbol, while the number of symbols in the training sequences depends on the number of active users $K$. In the noisy case, the sampling rate should be increased to make the algorithm robust to noise, however, the required sampling rate is typically far below the chip rate.

Clearly, we have decomposed the problem of multiuser channel estimation into a series of single user problems, where parameters of each user are estimated separately by considering only one corresponding column of the matrix C. Besides, unlike some high-resolution channel estimation methods that use iterative search and require a long training phase [5], our algorithm ensures a high channel throughput since the length of the training interval directly affects the channel capacity.

\section{SUBSPACE SIGNAL DETECTION}

The developed channel estimation algorithm is rather general and allows for implementation of different detection strategies, such as matched filters, multiuser detection schemes or RAKE receivers. In the following, we will discuss the application of our sampling results to the case of linear multiuser detection where all the operations are implemented on a reduced set of samples, obtained by sampling both the received signal and the reference with the same kernel and at the same rate.

\subsection{Linear Subspace Detector}

In quasi-synchronous CDMA systems, where a maximum delay spread of each user is relatively small compared to the symbol duration $T_{s}$, equation (4) can be directly used to solve for bits sent by each user, that is, without extracting individual channel parameters. During the training phase, the receiver should only estimate the matrix $\mathbf{C}$, while during the detection phase, bits sent by users $1,2, \ldots K$ are given by

$$
\mathbf{b}_{n}=\mathbf{C}^{-1} \cdot \mathbf{Y}_{n}
$$

It is clear that in this case the receiver does not require knowledge of the user's signature sequences, however, (6) can be successfully applied to systems where approximate timing of users is a priori known to the receiver. If that is not the case, the receiver must first estimate the unknown channel parameters and then compute a new matrix $\mathbf{C}_{d}$ that will be used for detection. The elements $c_{m k}$ of $\mathbf{C}_{d}$ are given by

$$
c_{m k}=S_{k}[m] \sum_{l=1}^{L}\left(a_{k}^{(l)} e^{-j m \omega_{0} \delta \tau_{k}^{(l)}}\right)
$$

where $\delta \tau_{k}^{(l)}$ now denotes a delay shift with respect to the new reference at the receiver, which is assumed to be on the order of a chip duration. The same approach can be extended to sequence detection, that is, we can solve directly for a sequence of bits $\mathbf{b}_{k}=\left[\begin{array}{llll}b_{k, 1} & b_{k, 2} \ldots & b_{k, L}\end{array}\right]$ sent by each user

$$
\mathbf{B}=\mathbf{C}_{\mathbf{d}}{ }^{-1} \cdot \mathbf{Y}
$$

The developed subspace detector is only one possible linear strategy that leads to error-free detection in the absence of noise, while the concept of subspace detection is more general and can be applied to other linear detection methods as well. For example, consider a decorrelating detector for a synchronous non-fading channel [8]. Assume that the received baseband signal $y(t)$ is sampled with the sinc sampling kernel and then passed through a bank of $K$ 
matched filters, each matched to the lowpass approximation of the corresponding signature waveform. The output of such a filter bank is given by

$$
\mathbf{y}_{\mathbf{o}}=\mathbf{R A b}+\eta
$$

where

$$
\begin{gathered}
\mathbf{A}=\operatorname{diag}\left(a_{1} a_{2}, \ldots, a_{K}\right) \\
\mathbf{b}=\left(b_{1} b_{2}, \ldots, b_{K}\right)
\end{gathered}
$$

while $\mathbf{R}$ is the normalized cross-correlation matrix of the filtered signature sequences $\widetilde{s_{k}}(t)$ and $\widetilde{s_{j}}(t)$, with elements $r_{k j}$ given by

$$
r_{k j}=\sum_{m} \widetilde{s_{k}}\left(m T_{s m p}\right) \widetilde{s_{j}}\left(m T_{s m p}\right)
$$

By multiplying the vector of matched filter outputs (9) by $\mathbf{R}^{-1}$, we get

$$
\mathbf{R}^{-1} \mathbf{y}_{\mathbf{o}}=\mathbf{A b}
$$

for $\sigma=0$. In order to recover transmitted bits, one can take the sign of each component in (11), that is,

$$
\hat{b_{k}}=\operatorname{sgn}\left(\mathbf{R}^{-1} \mathbf{y}_{\mathbf{o}}\right)_{k}=\operatorname{sgn}(\mathbf{A b})_{k}=b_{k}
$$

which achieves perfect detection for each active user in the absence of noise. The subspace detection is thus a simple and natural strategy that can be implemented in virtually any detection scheme, thus potentially allowing CDMA receivers to exhibit a good performance/complexity tradeoff.

\section{COMPUTATIONAL COMPLEXITY}

It is of interest to explicitly evaluate computational order of our developed scheme and compare it with that of the existing techniques.

The major computational requirement of the channel estimation method is dominated by the singular value decomposition part, which in the case of timing synchronization results in an overall computational order of $O\left(M^{3}\right)$ per user (in one synchronization frame), where $M$ denotes the number of samples per symbol. It is worth noting at this point that reducing the sampling rate $K_{s}$ times not only reduces the computational complexity, but also power consumption of A/D converters and the required processor speed, and that approximately by a factor of $K_{s}^{2}$.

Implementation of the simplest timing synchronization method based on matched filters requires $O\left(N^{2}\right)$ operations, where $N$ denotes the spreading factor. As we will see in the next section, the subspace estimator has essentially the same computational order, yet the technique based on matched filters does not have the satisfactory resolution performance and is suitable only for initial synchronization. High resolution methods [1] typically involve two computationally intense steps, namely, estimation of the $N \times N$ signal correlation matrix and the singular-valuedecomposition step, both requiring on the order of $O\left(N^{3}\right)$ computations.

We next focus on the computational complexity of developed subspace detectors. In the case of the subspace detection (6), the receiver must first compute a pseudoinverse of the matrix $\mathbf{C}$, which requires $O\left(M K^{2}\right)$ operations, while the detection phase itself has computational order of $O(M \log (M))$ per symbol (associated to computing $M$ DFT coefficients of the received signal). Similarly, implementation of the subspace decorrelating or MMSE linear detector involves inversion of a $K \times K$ matrix, a step that requires $O\left(K^{3}\right)$ computations, whereas the computational order during the detection phase is $O(M)$. This is clearly much lower than the order of the channel estimation part, yet the detection phase requires higher sampling rates to achieve performances comparable to those of chipsampled schemes, as we will see in the next section. Note that equivalent chip-sampled multiuser detection schemes require on the order of $O\left(K^{3}+N\right)$ operations per one detection frame.

\section{NUMERICAL RESULTS}

In this section, we show simulation results that illustrate the performance of the developed scheme. Figures 1-4 illustrate the timing synchronization performance of our method for an AWGN channel with BPSK modulation, where the spreading is achieved with pseudo-random sequences of length 511. Figure 1 shows an average timing estimation error (normalized to the chip duration $T_{c}$ ) versus the sampling rate in non-fading channels with 10 users. Our algorithm clearly yields high resolution estimates ${ }^{1}$ even with a modest number of samples. For example, by sampling the received signal at one fourth of the chip rate, the average estimation error is less than one tenth of the chip duration (for $E_{b} / N_{0} \geq 7 d B$ ). Comparison of the timing estimation errors for systems with 5 and 10 users is illustrated in Figure 2, indicating that the number of active users doesn't affect the estimator performance, since we have decomposed the multiuser estimation problem into a series of single-user equivalents. Due to the same reason, our scheme is near-far resistant, as shown in Figure 3. The performance of our estimator in multipath fading channels is illustrated in Figure 4, where we assumed 3 propagation paths for each user. The error is somewhat higher than in the case of non-fading channels, however, it is interesting to note that if there is only one dominant path, the estimation error is basically the same as in the non-fading channels.

\footnotetext{
${ }^{1}$ Within a fraction of the chip duration.
} 
We next consider the bit-error-rate performance of the developed subspace detection scheme in quasisynchronous systems, where timings of all users are assumed to be perfectly estimated. Figure 5 illustrates the performance in the system with 10 users versus the sampling rate, while dotted lines correspond to the decorrelating detector implemented on the same set of samples. The two methods have essentially the same performance, however, the BER exhibits a relatively slow decay rate. Namely, if the sampling rate is below the chip rate $R_{c}$, we lose a part of the signal energy and effectively reduce the SNR, which in turn increases the average BER. Note that the same argument holds for the timing estimation performance, however, in this case a dependence of the estimation error on the SNR is not the same. In Figure 6 we compare the detection performance of chip-sampled and subsampled schemes for different values of SNR. Roughly speaking, sampling at half the chip rate is sufficient to attain the average BER close to that of the chip-sampled scheme. Whether or not it is advisable to detect the signal from its subspace is obviously a matter of tradeoff between the complexity of the receiver on the one hand and its performance on the other, which is particularly relevant in the case when computational requirements and power consumption are the main constraints in the system design.

\section{CONCLUSION}

We have presented a new approach to the problem of designing low-complexity digital receivers for DS-CDMA systems, where all the necessary steps are carried out on a sampled lowpass version of the received signal. We extended some of our recent sampling results for certain classes of non-bandlimited signals to the multiuser channel estimation problem and developed a method that estimates the unknown channel parameters of all users simultaneously, and that from the uniform set of samples of the received signal taken well below the chip rate. Once the channel parameters of each user have been estimated, they are used in the detection process, carried out on a lowpass version of the received signal as well. Our approach leads to lower computational requirements and reduced power consumption compared to existing solutions, thus allowing for a practical hardware implementation and all the benefits of a digital design.

\section{REFERENCES}

[1] S. E. Bensley and B. Aazhang, "MaximumLikelihood Synchronization of a Single User CodeDivision Multiple-Access Communication Systems",

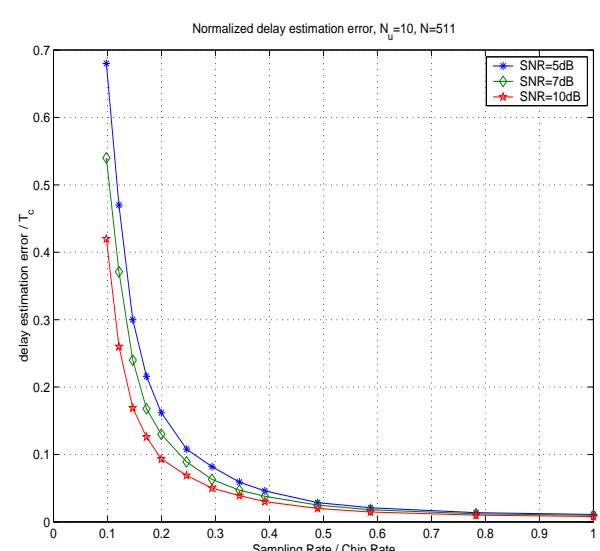

Fig. 1. Average timing synchronization error (normalized to $T_{c}$ ) in the multiuser case (10 users) vs. sampling rate. We assumed a non-fading channel. Signature sequences of all users are of length 511.

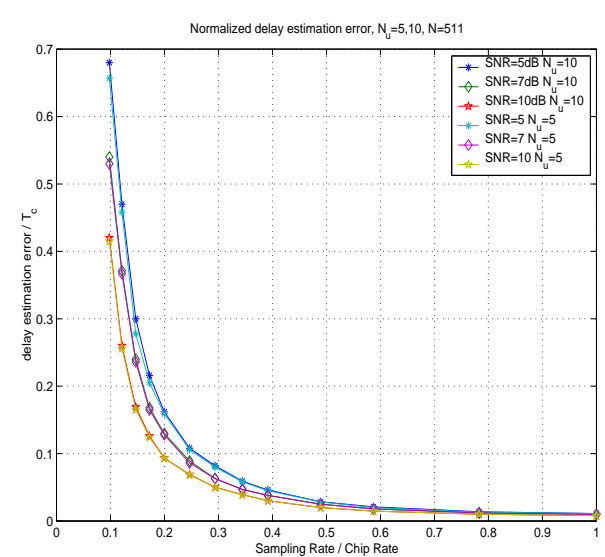

Fig. 2. Comparison of the timing synchronization errors in non-fading channels for systems with 5 and 10 users

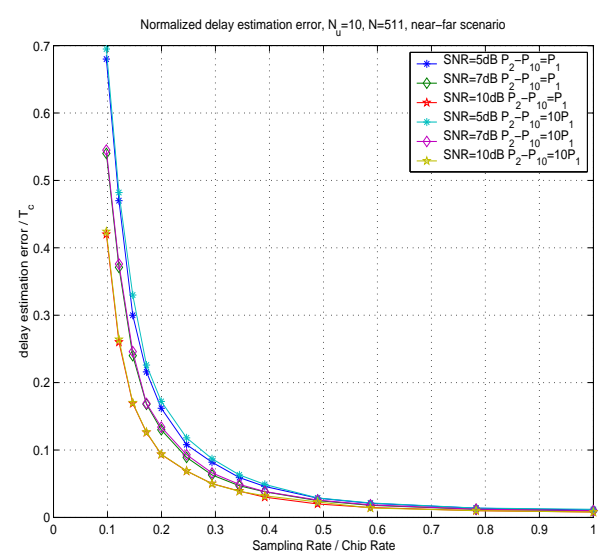

Fig. 3. Timing synchronization errors for a system with 10 users in the near-far case. 


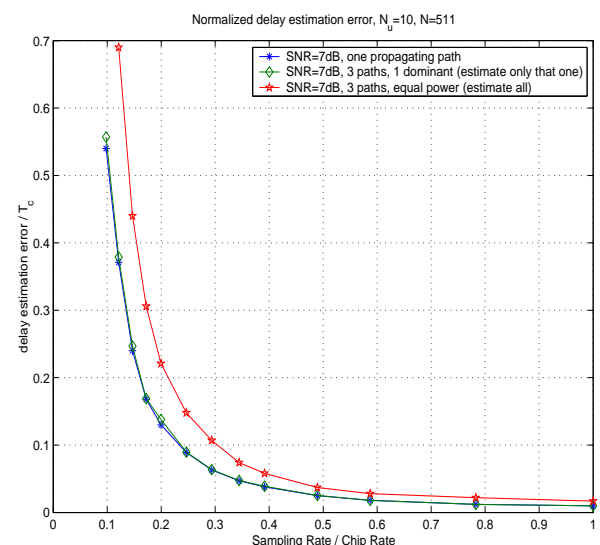

Fig. 4. Comparison of timing estimation errors for the system with 10 users is single-path and multipath channels (3 propagation paths for each user).

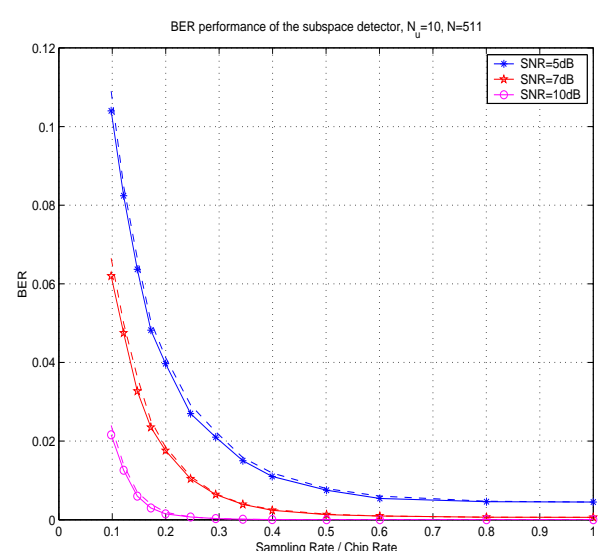

Fig. 5. BER performance of the subspace detector in non-fading synchronous CDMA systems with 10 users. Dotted lines illustrate the BER performance of the decorrelating detector.

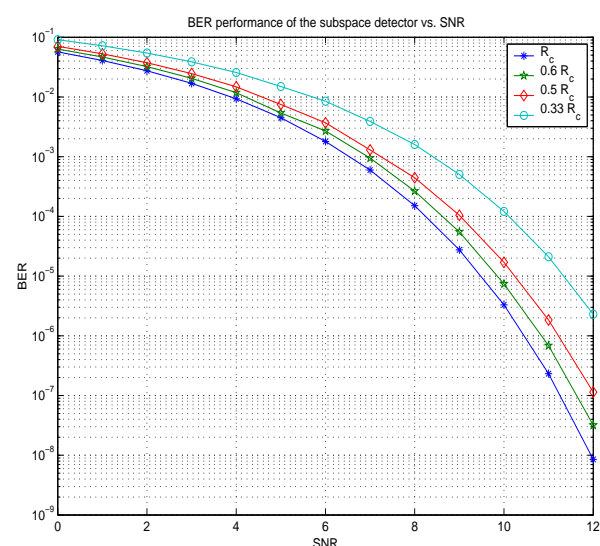

Fig. 6. BER performance of the subspace detector $v s$. SNR for different values of the sampling rate.
IEEE Transactions on Communications, Vol. 46, No. 3., p.p. 392-399, March 1998.

[2] S. Y. Kung, K. S. Arun and D. V. Bhaskar Rao, "State-space and singular-value decomposition-based approximation methods for the harmonic retrieval problem", Journal of the Optical Society of America, Vol. 73, No. 12, pp. 1799-1811, December 1983.

[3] Z. S. Liu, J. Li and S. L. Miller, "An efficient codetiming estimator for receiver diversity DS-CDMA systems", IEEE Transactions on Communications, Vol. 46, pp 826-835, June 1998.

[4] I. Maravic and M. Vetterli, "Sampling Results for Classes of Non-Bandlimited 2-D Signals", IEEE Transactions on Signal Processing, submitted December 2001.

[5] J. Ramos and M. Zoltowski, "Low-Complexity Space-Time Processor for DS-CDMA Communications", IEEE Transactions on Signal Processing, Vol. 48, No. 1, January 2000.

[6] B. D. Rao and K. S. Arun, "Model Based processing of Signals: A State Space Approach", Proceedings of the IEEE, Vol. 80, No. 2, pp. 283-309, February 1992.

[7] E. G. Strom, S. Parkvall, S. L. Miller and B. E. Ottersten, "DS-CDMA synchronization in time-varying fading channels", IEEE J. Selected Areas in Coтmunications, vol. 14, pp. 1636-1642, October 1996.

[8] S. Verdu, Multiuser Detection, Cambridge University Press, 1998.

[9] M. Vetterli, P. Marziliano and T. Blu, "Sampling signals with finite rate of innovation", IEEE Transactions on Signal Processing, June 2002.

[10] A. J. Viterbi, CDMA - Principles of Spread Spectrum Communication Reading, MA: Adison-Wesley, 1995. 